\title{
Review of: "Impact of KRAS mutation status on the efficacy of immunotherapy in lung cancer brain metastases"
}

JEAN-BAPTISTE ASSIE

Potential competing interests: The author(s) declared that no potential competing interests exist.

This is a monocentric retrospective study which evaluate the impact of KRAS mutation on the efficacy of ICI in NSCLC with brain metastasis (BM).

They showed an improvement of overall survival (from BM diagnosis) when ICl are used within the 90-days (ICl-90) of diagnosis versus chemotherapy. Moreover, in the subgroup of KRAS mutated patients, they showed a better overall survival with ICl-90 than chemotherapy.

However, they did not show a significant difference between KRAS mutated vs non-mutated in the ICI-90 regimen.

Since, the line of treatment is not indicated in the paper or in characteristic table, it is difficult to extrapolate the results of this study. In fact, nowadays all patients receive $\mathrm{ICl}$ in front-line regimen with or without chemotherapy. And if they don't receive $\mathrm{ICl}$ in 1st setting, they will have it in second line. Futhermore, there is a discrepancy with the survival in the ICl-90 regimen for KRAS status. Many studies showed that KRAS mutated patients will respond better than KRAS wild-type (recent meta-analysis presented at ASCO 2021). But maybe the impact of brain metastasis overcomes the KRAS status ?

This paper is relevant to tell us that if your patient is on second or more line of treatment and bear a KRAS mutation, it is better to offer him ICl regimen than chemotherapy. 